\title{
Computer Simulation for Ion Sources Optimization ${ }^{1}$
}

\author{
V.I. Gushenets and I.V. Litovko* \\ High Current Electronic Institute SB RAS, 2/3, Academichesky ave., Tomsk, 634055, Russia \\ *Institute of Nuclear Research NASU, 47, pr. Nauki, Kiev, 03028, Ukraine
}

\begin{abstract}
Results of the computer simulation for ion sources optimization used for ion implantations have been done. The highly stripped ion source has been designed to provide high current beams of multiply charged Phosphorous and Boron ions for high energy ion implantation. However, the total current transport efficiency was about $30 \%$. The modified computer code KOBRA has been used to simulate processes of ion extraction by dcacceleration systems as well as beam transport and thus to determine main reasons for ion beam losses. The calculations indicated that the losses of extracted ion beam mainly occur in the transport channel and magnetic separator. The computer modeling allows find optimal geometry for ionoptical system. Several ion-optical systems were designed and also changed the design of the initial section of the beam transport channel. Furthermore, the simulation for original way of compensating the parasitic beam deflection has been executed. Results of experiments with the modified geometry are supported simulation results. With the optimization of geometries of the ion-optical system and experimental setup, the maximum current transport for Boron ions has been attained. It should be noted that the maximum attainable percentage of singly charged $B$ ions was $65 \%$ and the total current transport was about $60 \%$.
\end{abstract}

\section{Introduction}

Various types of ions, but mostly Boron and Phosphorous are implanted into substrate used in the construction of semiconductors. The energies range deferent from as low as $100 \mathrm{eV}$ for shallow surface implantations, to as high as multi-MeV for deep implantation into materials. Our task was to develop high charge state ion sources for high energy implantation in order to improve upon present day high-energy ion implanters. The natural way for this purpose was trying to adapt charge enhancement techniques to ion sources that generate steady state multi-charged B, P ions [1].

The highly stripped ion source has been designed to provide high current beams of multiply charged Phosphorous and Boron ions for high energy ion implantation. However, the total current transport efficiency was about $30 \%$. Therefore, determine main reason for ion beam losses and optimization construction of ion-optical system was main tasks for improv- ing effective of ion source. The use of computer modeling for these purposes looks very attractive and easy way in order to optimize beam parameters as well as geometry of ion source.

Mathematically problem investigation of intensive charge beams come to solution of nonlinear selfconsistent system equations that have analytical solution in simplest cases only. Thus the main mathematical method of solving such tasks is numerical modeling. The aim of these simulations is always to investigate the charge particle beam quality, which makes optimization for a specific application possible. As a rule, the applied numerical method based on solving the Poisson equation with the unknown space charge term and then the result is used for the solving of motion equations for charge particles. A repeated iteration allows achieve self-consistent solution.

The optimization of the geometry of ion-optical system as well as transport system was made in this work by consequent numerical simulations with Kobra code [2]. Algorithms of code are modified for the calculation of the beam characteristics with the best precision.

\section{Mathematical model for numerical simulation of beam extraction}

Code Kobra is intended for solving three-dimension stationary problems of forming charged particle beams in external and self-consistent electric and magnetic fields. It allows translate the geometry information into mesh information and take into account plasma source geometry and acceleration gap geometry as well as physical condition for beam formation. The plasma is looking as collisionless, fully ionized, homogeneous density particle distribution is assumed. We consider the case of electron emission limited by space charge. For describing of such plasma model could be used Poisson equation $\Delta \varphi=\rho / \varepsilon$, the law of charge conservation $\nabla j=0$ and particles movement equation

$$
\frac{d v_{l}}{d t}=\frac{q_{i}}{m_{i}}\left(E+\left[v_{i} \times B\right]\right),
$$

here $\varphi$ is the electric potential, $E$ is the electric field, $B$ is the magnetic induction, $j$ is the current density, $\varepsilon$ is the permittivity, $v_{i}, m_{i}$, and $q_{i}$ are the velocity, mass and charge for particle of kind $i$. For transport high-

${ }^{1}$ The work was supported by the Russian Foundation for Basic Research (Grant No. 07-08-00337-a). 
current ion beam we need to take into account the importance the space charge of the particles in addition to the external fields

$$
\rho=\sum_{i=I}^{N} \frac{J_{i}}{v_{i}} .
$$

Here $J_{i}$ is the current density, $N$ is the maximum charge of ions in the beam and the magnetic self-field that may influence the particles themselves

$$
B=\mu_{0} \mu_{r} J / r
$$

where $\mu_{0} \mu_{r}$ is the permeability and $r$ is the perpendicular distance to the trajectory. The space charge limited current density $j$ depends on the potential drop $\varphi$ across the extraction gap width $d$ according to ChildLengmuir low

$$
J-\frac{4 \varepsilon_{0}}{9} \sqrt{\frac{2 q}{m}} \frac{\varphi^{3 / 2}}{d^{2}} .
$$

The beam is extracted from plasma by applying a potential difference between the plasma and the beam line.

The finite difference method (FDM) is used for the discretisation of equations system. The highest discretisation should be chosen to translate the physical problem into the best possible data description, but the Debye length

$$
\lambda_{D}=\sqrt{\frac{\varepsilon_{0} k T}{n_{e} q^{2}}}
$$

must be smaller than the character length of mesh discretization.

For solution of the set of algebraic equations an iterative point-to-point relaxation method is applied. The first step is solution of the Laplace equation with using seven point differential schemes. An iteration method with relaxation of potential is used to find the self-consistent solution:

$$
\varphi_{i, j, k}^{n+1}=\alpha \varphi_{i, j, k}+(1+\alpha) \varphi_{i, j, k}^{n}
$$

where $n$ is the number of iteration, $\alpha$ is the coefficient of relaxation that can change during calculation, $\varphi_{i, j, k}^{n}$ is the old value of potential, $\varphi_{i, j, k}^{n+1}$ is the new, $\varphi_{i, j, k}$ is the evaluated for node $(i, j, k)$ from calculation for this node and neighbored nodes.

Equations of motion is solving by numerical integration with repeated interval halving and extrapolation. The space charge map is created during this process. A self-consistent solution can be found by repeated solving of Poisson equation, motion equations for particles and re-determination of the space charge distribution on every step. The existing boundaries between regions with space charge and region with plasma condition are taken into account.

\section{Results of computer calculations}

Simulations have been made for plasma ion source with different geometry of ion-optical system. The principal scheme of it for ions extraction and acceleration is shown at Fig. 1.

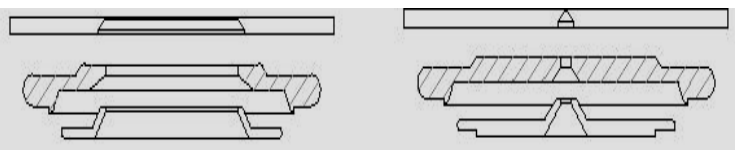

Fig. 1. Construction of ion-optical 3-electrodes system

The ac-dc system is used for saving the space charge compensation of the extracted ion beam. The beam is extracted from plasma by applying a potential difference between the plasma and the beam line. System is in magnetic field. The aperture sizes of electrodes as well as distances between them changed during calculation with aim to find optimal construction for extraction of the steady ion beam with high current, small divergence and minimizing losses during beam transport.

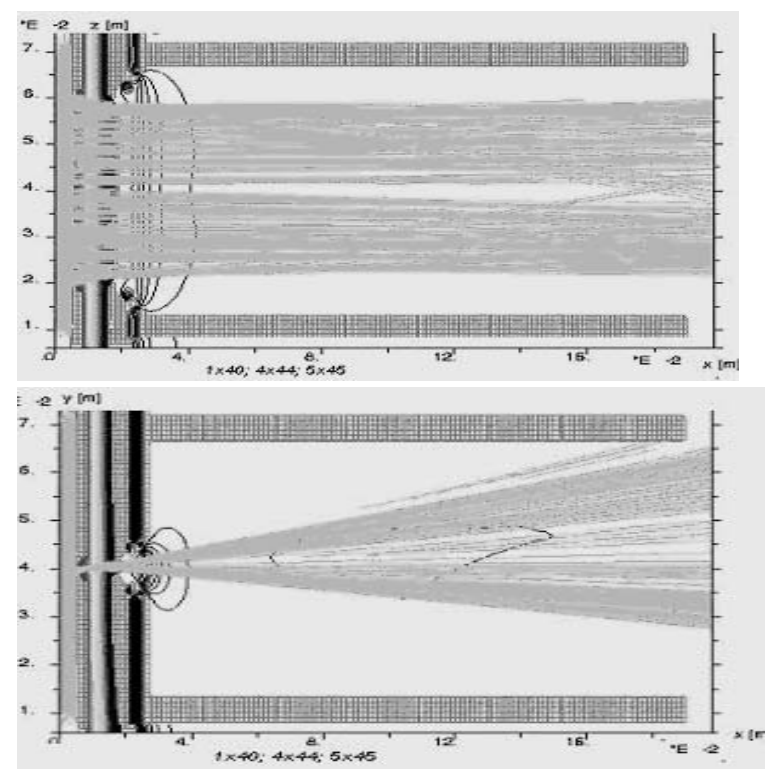

Fig. 2 Calculated trajectories of the ion beam along (upper) and across (bottom) emission slit for initial geometry: size of emission slit is $1 \times 40 \mathrm{~mm}$, suppressor and accel-electrode are of $4 \times 44$ and $5 \times 45 \mathrm{~mm}$ consequently

The calculation has been made for Phosphorus ion beam with different ion charge state $\left(45 \% \mathrm{P}^{+1}\right.$, $45 \% \mathrm{P}^{+2}, 10 \% \mathrm{P}^{+3}$ on current fractions) and for Boron beam $\left(70 \% \mathrm{~B}^{+}, 10 \% \mathrm{~F}^{+}, 10 \% \mathrm{BF}^{+}\right.$and $\left.10 \% \mathrm{BF}^{+2}\right)$. The starting energy is given by a direct ion drift energy which is determined by the physics of plasma formation and the ion temperature. Corresponding data for plasma source have been taken from experiment [3].

The space charge inside the plasma is compensated by electrons with Boltzmann density distribution and temperature of about $5 \mathrm{eV}$. Calculations have been made for emission current $20 \mathrm{~mA}$ and $40 \mathrm{~mA}$, ac- 
voltage $25 \div 30 \mathrm{kV}$ and dc-voltage $2 \div 6 \mathrm{kV}$. System was subdivided on 2 subsystems - extraction subsystem and transport subsystem. Fig. 2 shows the results of calculation for these subsystems for initial geometry.
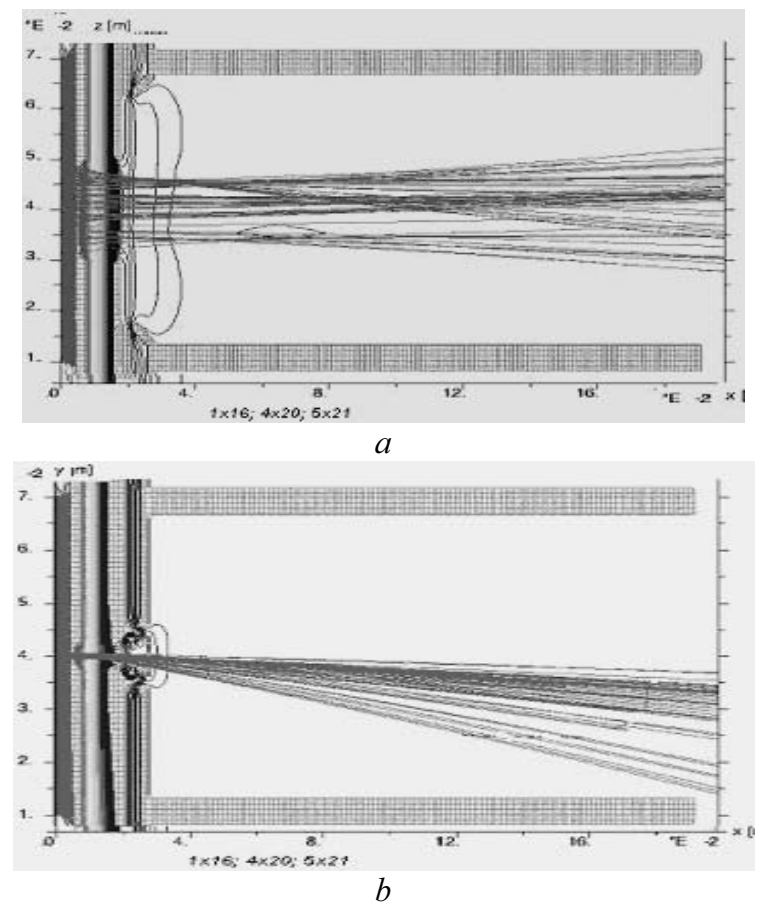

Fig. 3 Calculated trajectories of the ion beam along $(a)$ and across $(b)$ emission slit for aperture size in emission, ac- and dc-electrodes $1 \times 16,4 \times 20,5 \times 21 \mathrm{~mm}$. The beam de-

flected by the magnetic field of the ion source $(b)$

It can be seen from the Fig. 2 that part of beam losses on the walls of the first section due to deflection of the beam in the magnetic field of the ion source magnet and losses for the section make up $20 \div 30 \%$. To increase beam transparency, source aperture was reduced from $1 \times 40 \mathrm{~mm}$ to $1 \times 16 \mathrm{~mm}$ that shows best result under calculation, besides was reduced aperture size for ac- and dc-electrodes. Fig. 3 shows the result of calculation for reducing size.

Ones can see from Figs. 2 and 3 that reducing slits sizes allows decrease defocusing influence of these slits owing to reduce beam divergence and rejected beam losses on the tube walls. But problem connected with decreasing beam cross-sectional dimensions before the entrance aperture of the separator still exist. We need in beam cross-section at separator entrance not more than $4.8 \times 16 \mathrm{~cm}^{2}$, but we have total beam dimensions $8.5 \times 10 \mathrm{~cm}^{2}$ and the losses for a $4.5 \times 10 \mathrm{~cm}^{2}$ beam cross-section area make up $13 \%$.

In analyzing the calculation data, we came to the conclusion that the maximum decrease in beam crosssectional dimensions is attainable not only by decreasing the vertical dimension of the emission slit of the ion source, but also by reducing the spacing between the emission boundary and the entrance aperture of the separator. Decreasing the spacing by $20 \mathrm{~cm}$ must de- crease the cross-sectional dimensions by a factor of $>1.5$ even for an ion source with an emission slit of $1 \times 25 \mathrm{~mm}^{2}$. However in this case, we have to do away with the positioning unit and resolve the problem of adjusting the ion beam position, since the deflection of the ion beam in the self-magnetic field of the source should be compensated. From Fig. 3, $b$ we can see that the beam deflected by the magnetic field of the ion source. The displacement the suppressor and grounded electrode slits from the emission slit by $1 \mathrm{~mm}$ in the direction of beam deflection may compensate beam deflection. As a result, the ion beam shifted to the slit edge and came under the influence of the transverse electric field, which causes deflection of the beam ions in a direction perpendicular to the beam motion and opposite to the deflection produced by the magnetic field of the ion source. Fig. 4 shows results of calculation for shifted construction.
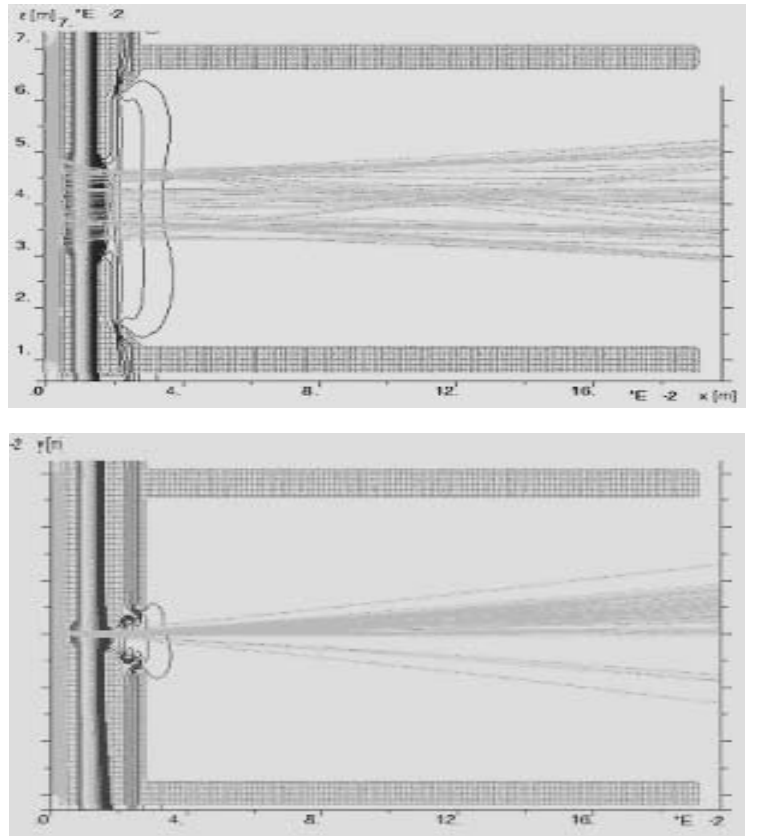

Fig. 4 The beam deflection is compensated through displacing the suppressor and the grounded electrode downwards by $1 \mathrm{~mm}$

Thus displacing the suppressor and the grounded electrode downwards by $1 \mathrm{~mm}$ allows us to reduce the spacing between the emission boundary and the separator about $20 \mathrm{~cm}$ and as result greatly decreased beam cross-sectional dimension. Calculations showed that the total beam dimensions were decreased down to $4.2 \times 6 \mathrm{~cm}^{2}$. Actually the vertical dimension of the beam became much smaller and was no greater than $2 \mathrm{~cm}$.

The results of calculations support the experimental data. This way of compensating the beam deflection has a number of shortcomings, among which is an increase in horizontal beam dimension; however, this dimension was found to approximate the calculated one. 


\section{Experimental results}

Two ion-optical systems were designed, of which one had an emission slit of $1 \times 25 \mathrm{~mm}^{2}$ and the other an emission slit of $1 \times 16 \mathrm{~mm}^{2}$. We also changed the design of the initial section of the beam transport channel, which allowed an increase in the inner diameter of the accel-electrode from 57 to $74 \mathrm{~mm}$. Thus, the conductivity in the initial section was doubled, and hence the pressure inside it was to decrease twofold. Furthermore based on the calculations of the compensating beam deflection way we have revised our experimental set up and removed the bellows unit whereby the ion beam position in the entrance aperture plane of the separator beam line was changed. This made it possible to reduce the spacing between the emission boundary and the separator by $17 \mathrm{~cm}$.

The cross section of the ion beam and its position in the entrance aperture region of the magnetic separator beam line were determined from an imprint left on the molybdenum collector plate. Fig. 5 shows a photo of the imprint. An ion beam of current irradiated the Mo plate for an hour. The pressure at the collector site was $9.2 \times 10^{-5}$ Torr.

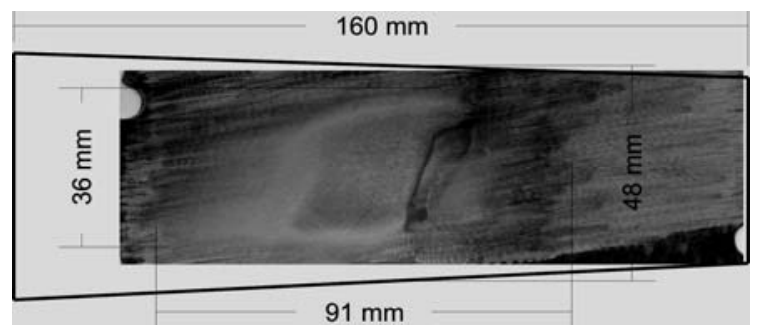

Fig. 5. Boron ion beam imprint on the molybdenum collector plate against the background of the beam line contour of the separator for the source aperture is $1 \times 25 \mathrm{~mm}^{2}$

It can be seen in the figure that the cross-sectional dimensions of the beam, particularly its vertical dimension, were some decreased and were no more than $36 \times 91 \mathrm{~mm}^{2}$. This measuring procedure does not ensure sufficient accuracy, and hence the above values differ from those calculated using the computer code COBRA (Fig. 4) which shows that the full beam dimensions are $8.5 \times 10 \mathrm{~cm}^{2}$ and the for $4.5 \times 10 \mathrm{~cm}^{2}$ beam dimensions make up $13 \%$.

Moreover, the electrodes of the ion-optical system with an emission slit of $1 \times 16 \mathrm{~mm}^{2}$ were made. Calculations showed that the total beam dimensions were decreased down to $4.2 \times 6 \mathrm{~cm}^{2}$. Actually the vertical dimension of the beam became much smaller and was no greater than $2.5 \mathrm{~cm}$. Under different experimental conditions, the current transport varied between $50 \%$ and $60 \%$.

\section{Conclusion}

For optimal ion source parameters, beams more $40 \mathrm{~mA}$ were extracted. Singly charged boron made up over $70 \%$ of the total ion beam [3]. Due to the very limited acceptance of the bending magnet, only about $25 \div 35 \%$ of the beam can reach the collector. To increase beam transparency, the experimental set up have been redesigned and source aperture was reduced from $1 \times 40 \mathrm{~mm}^{2}$ to $1 \times 16 \mathrm{~mm}^{2}$. In results this arrangements allowed the full current transport of boron ion beam to increase practically twofold.

\section{Acknowledgments}

It is indeed a pleasure to thank Ady Hershcovitch, Efim Oks and Timur Kulevoy for many fruitful and inspiring discussions, and Alex Bugaev for help in experiments.

This work was supported by the U.S. Department of Energy, Office of Nonproliferation and International Security, Initiatives for Proliferation Prevention, Project No. IPP-BNL-T2-10226.

\section{References}

[1] V.A. Batalin, A S.Bugaev, V.I.Gushenets et al., Rev. Sci. Instrum. 75, 1900 (2004).

[2] I.G. Brown, The physics and technology of ion sources, Weinheim, Wiley-VCH Verlag GmbH \& Co. KGaA, 1999, pp. 41-60.

[3] V.I. Gushenets, A.S. Bugaev, E.M. Oks, A. Hershcovitch, B.M. Johnson, T.V. Kulevoy, H.J. Poole, and A.Y. Svarovski, Rev. Sci. Instrum. 77, 109 (2006). 